\title{
Knowledge, Attitudes, and Oral Health Practices of School Children in Davangere
}

\author{
Satish Vishwanathaiah
}

\begin{abstract}
Aims: The aim of this study was to assess the knowledge, attitude, and behavior of school children towards oral health. Settings and design: Descriptive study.

Materials and methods: School children $(n=700)$ aged between 10 to 14 years in a Davangere school were recruited into this study. The subjects completed a questionnaire that aimed to evaluate young school children's behavior, knowledge, and perception of their oral health and dental treatment.

Statistical analysis: The results were statistically analysed and percentage was calculated.

Results and conclusion: The participant oral hygiene habits (such as tooth brushing) were found to be irregular, and parent role in the oral hygiene habits of their children was limited. The study population showed higher awareness of caries than periodontal conditions. The children in this study also recognized the importance of oral health. The results of this study indicate that Comprehensive oral health educational programs for both children and their parents are required to achieve this goal.
\end{abstract}

Keywords: Attitude, Knowledge, Oral hygiene, Practice, School children

How to cite this article: Vishwanathaiah S. Knowledge, Attitudes, and Oral Health Practices of School Children in Davangere. Int J Clin Pediatr Dent 2016;9(2):172-176.

Source of support: Nil

Conflict of interest: None

\section{INTRODUCTION}

The American Dental Association recommends that to avoid oral diseases individual should brush and floss at least once a day and visit a dentist regularly. ${ }^{1}$ We all know that lack of oral hygiene practices leads to development of oral diseases. These practices, such as brushing, flossing, and periodic dental visit should be developed early in the childhood. Dental flossing and tooth brushing are the most commonly performed oral self-care behavior. ${ }^{2}$ About one-third of the sample reported flossing at least once a day. Three quarters of the population reported making periodic dental visits at least once a year. ${ }^{3}$ Most

\section{Associate Professor}

Department of Pedodontics, College of Dentistry, Jazan, Jazan University, Kingdom of Saudi Arabia

Corresponding Author: Satish Vishwanathaiah, Associate Professor, Department of Pedodontics, College of Dentistry Jazan, Jazan University, Kingdom of Saudi Arabia, Phone: +966504188075, e-mail: drvsatish@rediffmail.com of the children (73-83\%) in Sweden, Denmark, Germany, Austria, and Norway brushed their teeth twice a day. The use of the dental floss was rare. In general, flossing was less frequent among boys than among girls. ${ }^{4}$ Recent studies carried out in Michigan have found that, on an average, subjects reported brushing their teeth twice daily. Around $90 \%$ of the population brushed at least once a day. African-Americans are less likely than whites to brush and floss thoroughly and get regular dental checkup. ${ }^{5}$

So this study was done to assess the knowledge, attitude, and behavior of school children toward oral health in Davangere population.

\section{MATERIALS AND METHODS}

A total of 700 school children in the age group of 10 to 14 years were included in this study. Permission from the concerned authorities was taken in prior. Before and after the survey procedure, all the student were subjected to Oral Hygiene Index -Simplified (OHI-S) and assessed. In order to assess knowledge, attitude, and practice regarding oral health, a questionnaire ${ }^{6}$ having 22 questions was prepared for the school children. With the permission from the dean of the school, dental health questionnaire was explained to the students. Later, the questionnaire was distributed among the children in the classrooms at the starting of the session and collected at the end of the session.

\section{RESULTS}

The results were tabulated, percentage was calculated, and conclusions were drawn. The survey presented a comprehensive overview of the knowledge, attitudes, and practices of school children aged 10 to 14 years in a Davangere school.

Table 1 shows that $70.42 \%$ of the children showed poor oral hygiene prior to the study. After the survey, OHI-S

Table 1: Simplified Oral Hygiene Index

\begin{tabular}{llll}
\hline & \multicolumn{2}{c}{ Before } \\
\cline { 2 - 4 } & Total & $\%$ \\
\hline Good & 15 & & 2.14 \\
Fair & 183 & & 26.14 \\
Poor & 493 & After \\
\hline & \multicolumn{2}{c}{} \\
\cline { 2 - 4 } & Total & $\% 2.57$ \\
\hline Good & 438 & 27.57 \\
Fair & 193 & 9.85 \\
Poor & 69 & \\
\hline
\end{tabular}


Table 2: Knowledge toward oral hygiene by school children in a Davangere school

\begin{tabular}{|c|c|c|}
\hline \multicolumn{3}{|c|}{ K1. Has oral health got any role on general health? } \\
\hline & Total & $\%$ \\
\hline a. Yes & 700 & 100 \\
\hline b. No & - & - \\
\hline c. Don't know & - & - \\
\hline \multicolumn{3}{|c|}{ K2. What does irregular tooth brushing cause? } \\
\hline & Total & $\%$ \\
\hline a. Decay & 327 & 46.80 \\
\hline b. Gum disease & 65 & 9.20 \\
\hline c. Bad breath & 56 & 8 \\
\hline d. Stains on teeth & 131 & 18.8 \\
\hline e. Nothing & - & - \\
\hline f. All of the above & 121 & 17.20 \\
\hline \multicolumn{3}{|l|}{ K3. Why do we get dental problems? } \\
\hline & Total & $\%$ \\
\hline a. Eating sweets and ice creams & 448 & 64 \\
\hline b. Not brushing properly & 129 & 18.42 \\
\hline c. Not rinsing the mouth & 6 & 0.85 \\
\hline d. Not regularly visiting a dentist & 14 & 2 \\
\hline e. All of the above & 103 & 14.71 \\
\hline \multicolumn{3}{|l|}{ K4. How can we prevent dental problems? } \\
\hline & Total & $\%$ \\
\hline a. Avoiding sweets and sticky foods & 338 & 48.28 \\
\hline b. Brushing properly & 57 & 8.14 \\
\hline c. Mouth rinsing after meals & 3 & 0.43 \\
\hline d. Regularly visiting a dentist & 19 & 2.72 \\
\hline e. All of the above & 283 & 40.43 \\
\hline
\end{tabular}

K5. Do you know that clean mouth can prevent tooth decay?

$\begin{array}{lll} & \text { Total } & \% \\ \text { a. Yes } & 674 & 96.29 \\ \text { b. No } & 26 & 3.71\end{array}$

K6. Do you know that a dentist can clean and polish your teeth?

$\begin{array}{lll} & \text { Total } & \% \\ \text { a. Yes } & 700 & 100 \\ \text { b. No } & - & -\end{array}$

K7. What can be prevented by regular cleaning of mouth?

$\begin{array}{lll} & \text { Total } & \% \\ \text { a. Bleeding from gums } & 50 & 7.14 \\ \text { b. Loosening of gums } & 44 & 6.29 \\ \text { c. Loss of teeth } & 3 & 0.43 \\ \text { d. Bad smell } & 405 & 57.86 \\ \text { e. All of the above } & 198 & 28.28\end{array}$

analysis indicated that $62 \%$ of the children improved and showed good oral hygiene.

In this part of the survey, (Table 2) knowledge toward oral hygiene was measured. All children demonstrated that oral health had significant role in general health.

According to this, $46.80 \%$ of children said that decay is caused due to irregular brushing, whereas $9.2 \%$ children accepted that irregular tooth brushing causes gum diseases, and $8 \%$ of children concluded that irregular tooth brushing causes bad breath. Around $17.20 \%$ of children informed that all the factors were responsible for irregular tooth brushing.
Table 3: Attitude toward oral hygiene by school children in a Davangere school

\begin{tabular}{|c|c|c|}
\hline \multicolumn{3}{|c|}{$\begin{array}{l}\text { A1. Do you think maintaining healthy mouth is an individual } \\
\text { responsibility? }\end{array}$} \\
\hline & Total & $\%$ \\
\hline a. Yes & 696 & 99.43 \\
\hline b. No & 4 & 0.57 \\
\hline \multicolumn{3}{|c|}{ A2. Have you visited a dentist before? } \\
\hline & Total & $\%$ \\
\hline a. Yes & 328 & 46.86 \\
\hline b. No & 372 & 53.14 \\
\hline \multicolumn{3}{|c|}{ A3. If yes, then for what reason? } \\
\hline & Total & $\%$ \\
\hline a. Decay & 156 & 22.29 \\
\hline b. Pain & 68 & 9.71 \\
\hline c. Filling & 23 & 3.29 \\
\hline d. Extraction & 19 & 2.71 \\
\hline e. Any others specify & 62 & 8.86 \\
\hline \multicolumn{3}{|c|}{$\begin{array}{l}\text { A4. Do you think it is required to visit a dentist periodically to } \\
\text { maintain the oral health? }\end{array}$} \\
\hline & Total & $\%$ \\
\hline a. Yes & 683 & 97.57 \\
\hline b. No & 17 & 2.43 \\
\hline
\end{tabular}

About $18.42 \%$ of children complained that dental problem occurs due to improper brushing. Around 64\% attributed eating fruits and ice cream as causative factors, and $14.7 \%$ of children accepted that all factors contribute for development of a dental problem. Only $2 \%$ children said that dental problems occur because of not visiting the dentist regularly.

About $8.14 \%$ children informed that by proper brushing method, dental problem can be prevented, and $48.28 \%$ children accepted the fact that by avoiding sweets and sticky food dental problems can be resolved. Only $0.43 \%$ of children said that by rinsing mouth after meals dental problems can be reduced.

Around $96.29 \%$ of children accepted that cleaning can prevent tooth decay, and $3.71 \%$ of children contradicted it. About $57.86 \%$ children said that bad smell can be controlled by regular cleaning of mouth, and 7.14 and $6.29 \%$ children accepted that regular cleaning of mouth control bleeding gums and loosening of gums respectively. Around $28.28 \%$ of children accepted that regular cleaning of mouth can prevent all the causative factors. All children knew that dentist can clean and polish their teeth.

In this section of the survey, (Table 3) attitude of the school children was measured.

About $99.43 \%$ of children knew that maintenance of healthy mouth is each individual responsibility. Around $46.86 \%$ of children had visited the dentist, and $53.14 \%$ of children hadn't visited the dentist before. It was noted that $22.29 \%$ of children visited dentist for decay reason, $9.71 \%$ for reason of pain, $3.29 \%$ constituted for filling, and $2.71 \%$ for extraction. Around $8.86 \%$ of children 
Table 4: Practice toward oral hygiene by school children in a Davangere school

P1. How do you clean your teeth?
a. Toothbrush and toothpaste
b. Toothbrush and tooth powder
c. Finger and tooth powder
d. Neem sticks
e. Any other specify

$P 2$. How often you clean your teeth?
a. Once daily
b. Twice daily
c. More than twice daily
d. After every meal

P3. How do you brush your teeth?

$\begin{array}{lll}\text { a. Use horizontal strokes } & 208 & 29.71 \\ \text { b. Use vertical strokes } & 48 & 6.86 \\ \text { c. Both in horizontal and vertical directions } & 350 & 50 \\ \text { d. Circular strokes } & 94 & 13.43\end{array}$

P4. How often you change your brush?

$\begin{array}{lll}\text { a. Once in } 3 \text { months } & 216 & 30.86 \\ \text { b. Once in } 6 \text { months } & 304 & 43.43 \\ \text { c. Yearly once } & 4 & 0.57 \\ \text { d. When bristles get frayed up } & 155 & 22.14 \\ \text { e. Don't know exactly } & 21 & 3\end{array}$

P5. What amount of paste you apply on your brush?

$\begin{array}{lll}\text { a. Full length of bristles } & 374 & 53.43 \\ \text { b. Half-length of bristles } & 249 & 35.57 \\ \text { c. Pea-sized amount } & 77 & 11\end{array}$

P6. Do you press the paste in between the bristles?

$\begin{array}{lll} & \text { Total } & \% \\ \text { a. Yes } & 269 & 38.43 \\ \text { b. No } & 431 & 61.57\end{array}$

P7. Do you rinse your mouth after meals?

$\begin{array}{lll}\text { a. Yes } & 24 & 3.43 \\ \text { b. No } & 646 & 92.29 \\ \text { c. Sometimes } & 30 & 4.28\end{array}$

P8. Do you clean your tongue?

$\begin{array}{lll}\text { a. Yes } & 694 & 99.14 \\ \text { b. No } & 6 & 0.86\end{array}$

P9. How do you clean your tongue?

\begin{tabular}{lll} 
a. Tongue cleaner & Total & $\%$ \\
b. Fingers & 553 & 79 \\
c. Toothbrush & 141 & 20.14 \\
d. Any others specify... & - & - \\
\hline
\end{tabular}

visited for other reasons like cleaning (oral prophylaxis) and a few for orthodontic consultation. Around $97.57 \%$ of children knew that periodic dental visit is required for maintaining good oral health. Only $2.43 \%$ children were unaware of it.
In this section of survey, (Table 4) practice toward oral hygiene was measured. According to this all children used toothbrush and toothpaste for cleaning the oral cavity. Almost $69.57 \%$ of children brushed their teeth once daily and $30.43 \%$ children brushed twice. About $29.71 \%$ children brushed teeth with horizontal strokes, $6.86 \%$ children brushed with vertical strokes, and $50 \%$ of children brushed both in horizontal and vertical direction. Only $13.43 \%$ children used circular strokes for brushing.

Around $43.43 \%$ of children changed their toothbrush once in 6 months, and $30.86 \%$ children changed toothbrush every 3 months. Only $0.57 \%$ children changed toothbrush once a year, and $22.14 \%$ changed toothbrush when bristles frayed up. About $3 \%$ of children were unaware of the time of changing the toothbrush. Around $53.43 \%$ children applied toothpaste to full length of bristles, $35.57 \%$ applied to half-length of the bristles, and $11 \%$ of children applied pea-sized amount of toothpaste. Only $38.43 \%$ applied the paste in between the bristles where as $61.57 \%$ children didn't apply the paste in between the bristles.

Around $92.29 \%$ children did not rinse the mouth after meals, $3.43 \%$ did rinse the mouth regularly, and $4.28 \%$ did rinse mouth sometimes. Again, $99.14 \%$ children did clean their tongue, and only $0.86 \%$ did not clean the tongue. About $79 \%$ of children did use the tongue cleaner for cleansing their tongue, and $20.14 \%$ of children used their finger to clean their tongue.

\section{DISCUSSION}

Oral health is an integral component of general health. Childhood is the age where children develops reflexes to maintain general hygiene practices and attitude toward health. Children can be ruptured well for their general and oral health. ${ }^{7}$ It is the duty of parents, teachers as well as children themselves to know the importance of oral hygiene. We can target children for educating and motivating them for oral health maintenance and awareness. It is important to start oral health education in their regular curriculum at school level. This will help not only in creating awareness but also in development of correct oral health practices thereby to control orodental problems. In many countries, a large number of children and parents have limited knowledge on the causes and prevention of the most common oral diseases. ${ }^{8}$ This paper focuses on knowledge, attitude, and practices of school children toward the oral health. The school children showed very good response toward the study.

The OHI-S recorded showed that prior to the study, $70 \%$ of the children had poor oral hygiene. At the end of the study, there was a tremendous change in the children knowledge, attitude, and practice toward the oral health. 
The study showed that children attitude toward the oral hygiene should be guided properly by the parents and the guardians as well as the school dental health programs. ${ }^{9}$ To have an impact on attitude and practices, children may take more time, but in the long term it will have positive effects. Similar results are seen in the studies by Petersen and Torres. $^{10}$

While assessing knowledge, children thought that irregular brushes cause only decay (46.80\%), but they were less unaware of gum diseases and bad breath. This showed that around $64 \%$ of children commented that dental problems were caused due to eating ice creams only; however, other causes like improper brushing methods, not rinsing the mouth, and irregular visit to dentist were little cited by the children. And $48.28 \%$ children cited that dental problems can be prevented by avoiding sweets and sticky foods, whereas other prevention technique like proper brushing methods, rinsing mouth after meals, and regular dental visit were lesser known. These results indicated that improvement in knowledge toward learning proper brushing technique is needed. Interventions to increase the knowledge, regular visit to the dentist, subsequent use of flossing are essential and are in argument with other studies. ${ }^{9}$

While assessing the attitude of the school children, most of the children knew that the individual has prime responsibility toward maintaining healthy mouth and also that periodic dental visit is required to maintain oral health. Only $46.86 \%$ children had visited dentist, among which $22.29 \%$ visited for the reason of decay. Almost $53 \%$ children did not visit the dentist. This may be due to fear of dental setup, ${ }^{11}$ lack of toothache, or lack of parental encouragement. ${ }^{12}$ We also agree with the fact stated by Jalevik et al ${ }^{13}$ in 1999 that lack of parents' regular dental attendance might be reflected in children dental attitude. The behavior displayed by parents might also be the cause of lack of attendance regarding visit to the dentist. Barker and Horton showed that delay in seeking dental care could be attributed to other factors like parental belief and practices, lack of economic resources, and accessibility of dental services. They also showed that parents play a vital role in influencing child's oral health. Other factors, such as extraction and oral prophylaxis were not known to the patients. This concludes the fact that dental caries is one of the most common diseases in children. Other conditions like gingival and periodontal conditions, oral hygiene maintenance, orthodontic treatment was least considered. Lack of both parental and child oral health education might also explain these findings. ${ }^{9}$ So more educational program and importance of maintaining oral health to motivate children as well as the parents is required.
While assessing the oral hygiene practices of the children, all children used toothbrush and toothpaste as oral hygiene aids, as reported elsewhere. ${ }^{14-17}$ About $99.14 \%$ of children were aware of tongue cleansing methods out of which 79\% children used tongue cleaner. Only 30.43\% of children bushed twice daily pertaining to the fact that parental guidance toward oral health education is still lacking. Survey found that high percentage of children brushed their teeth once daily only. The same results were seen in the study done by Al-Omari MK et al 2006 in North Jordan. ${ }^{12}$ Lack of both parental and child oral health education might explain these findings. ${ }^{18}$ The other reasons may be due to the age of our children who were included in the survey that they try to achieve independence and start their attempts to build their own identity without family interference. Lack of both parental and child oral health education may explain this. ${ }^{13}$ Out of 700 school children, only $13.43 \%$ of children was aware of circular strokes used while brushing (Fones technique). More than $50 \%$ of children were not knowing about changing of the toothbrush at regular intervals, that is, once in 3 months. Only 53.43\% children did apply toothpaste to length of bristles, and $38.43 \%$ children did press the toothpaste between the bristles. These factors correlate the fact that parental and school dental health guidelines were still lacking in the children. ${ }^{18}$ Regular school health program/education explaining these should be advised.

The oral health knowledge, attitude, and practice among the secondary school students are still below the satisfactory level. This awareness on importance of oral health should be motivated. As stated by Al-Omari MK et $\mathrm{al}^{12}$ we also accept that there is a need to decrease dependency on oral health personnel and encourage students to take responsibilities for their own oral health. As children spend their entire day in the school, schools are the advisable platform for educating, promoting oral health care. At this age, children are receptive to guidance and familiar with learning environment and culture. It is very important to target oral health education to the children since the lifestyle and hygiene practices once established at an early age can go a long way in spending rest of the life in a healthy way. ${ }^{19}$ It is important to include oral health education into school curriculum so that it is taken as a part of life. Countries like Australia and New Zealand have a very well-developed school oral health education program and have demonstrated decline in dental caries among school children in the last few decades. $^{20}$

From our study we conclude:

- Oral health education programs has to be conducted in all the school in large scale, and even parents should also be a part of such educational programs. 
- Parents' education is a must and should be included to promote preventive oral care. It can be achieved if school authorities permit to educate them on parent teacher meeting.

- Teachers should be given education so that they can impart knowledge and importance on oral health and its maintenance.

- Oral preventive care should be included in school curriculum so that children and teachers know the importance of the same.

\section{REFERENCES}

1. Hayward RA, Meetz HK, Shipiro MF, Freeman HE. Utilisation of dental services 1986 patterns and trend. J Public Health Dent 1989 Summer;49(3):147-152.

2. Gilbert GH, Duncan RP, Crandall LA, Heft MW, Ringelberg ML. Attitudinal and behavioural characteristics of older Floridans with tooth loss. Community Dent Oral Epidemiol 1993 Dec;21(6):384-389.

3. Ronis DL, Lang WP, Farghaly MM, Passow E. Tooth brushing, flossing, and preventive dental visits by Detroit area residents in relation to demographic and socioeconomic factors. J Public Health Dent 1993 Summer;53(3):138-145.

4. Kuusela S, Honkala E, Kannas L, Tynjälä J, Wold B. Oral hygiene habits of 11-year-old school children in 22 European countries and Canadian 1993/1994. J Dent Res 1997 Sep;76(9): 1602-1609.

5. Ronis DL, Lang WP, Antonakos CL, Borgnakke WS. Preventive oral health behaviour among African-Americans and whites in Detroit. J Public Health Dent 1998 Summer;58(3):234-240.

6. Raju HG, Nagesh L, Deepa D. Oral health promotion and intervention activities carried out in rural areas of Davangere district. A report on GOI-WHO Collaborative Programme, Vol. 7; 2006.

7. Petersen PE, Torres AM. Preventive oral health care and health promotion provided for children and adolescents by the municipal dental health service in Denmark. Int J Paediatr Dent 1999 Jun;9(2):81-91.
8. Parkash H, Duggal R, Mathur VP, investigators. Oral health module for prevention of dental caries in school children. A GOI-WHO-India Biennium Project, New Delhi, India; 2004.

9. Farsi JM, Farghaly MM, Farsi N. Oral health knowledge, attitude and behaviour among Saudi school students in Jeddah city. J Dent 2004 Jan;32(1):47-53.

10. Petersen PE, Danila I, Samoila A. Oral health behaviour, knowledge and attitudes of children, mothers and school teachers in Romania. Acta Odonto Scand 1995 Dec;53(6):363-368.

11. Taani DQ. Dental attendance and anxiety among public and private school children in Jordan. Int Dent J 2002 Feb;52(1): 25-29.

12. Al-Omari MK, Al-Wahadni MA, Saeed KN. Oral health attitudes, knowledge and behaviour among school children in north Jordan. J Dent Educ 2006 Feb;70(2):179-187.

13. Jalevik B, Sjostrom O, Noren JG. Evaluation of three years of dental care of adolescents in the public dental service in west Sweden. Sweden Dent J 1999;23(4):141-148.

14. Russel BA, Horowitz AM, Frazier PJ. School based preventive regiments and oral health knowledge and practices of sixth graders. J Public Health Dent 1989 Fall;49(4):192-200.

15. Lin HC, Wong MC, Wang ZJ, Lo EC. Oral health knowledge attitudes and practices of Chinese adults. J Dent Res 2001 May;80(5):1466-1470.

16. Al-Sadhan SA. Oral health practices and dietary habits of intermediate school children in Riyadh, Saudi Arabia. Saudi Dent J 2003 May-Aug;15(2):81-87.

17. Al-Ansari JM, Al-Jairan LY, Gillespie GM. Dietary habits of the primary to secondary school population and implications for oral health. J Allied Health 2006 Summer;35(2):75-80.

18. Rajab LD, Petersen PE, Bakaeen G, Hamdan MA. Oral health behaviour of school children and parents in Jordan. Int J Pediatr Dent 2002 May;12(3):168-176.

19. World Health Organisation. The status of School health. Report of the school health working group and the WHO expert committee on comprehensive school health education and promotion. Geneva: WHO; 1996.

20. Petersen PE. The world oral health report 2003: continuous improvement of oral health in the 21st century- the approach of the WHO Global Oral Health Programme. Community Dent Oral Epidemiol 2003 Dec;31 (Suppl 1):3-24 